\title{
Propuesta de modelo de gestión para disminuir la deserción de estudiantes de un instituto técnico superior de comunicaciones en el Perú
}

\section{Proposal for a management model to reduce the desertion of students from a higher technical institute of communications in Peru}

\author{
Marleni Dorothy Ruiz-Loayza \\ Universidad Norbert Wiener, Lima, Perú \\ nilemarizru@gmail.com \\ (iD https://orcid.org/0000-0002-7356-2915 \\ Raúl Valdiviezo- López \\ Universidad Norbert Wiener, Lima, Perú \\ raul.valdiviezo@uwiener.edu.pe \\ iD https://orcid.org/0000-0003-4670-3353 \\ Roy Fernando Martínez-Quintanilla \\ Universidad Norbert Wiener, Lima, Perú \\ roy.martinez@uwiener.edu.pe \\ (i) https://orcid.org/0000-0002-4223-0272
}

Recepción: 30/09/2021 | Aceptación: 15/12/2021 | Publicación: 10/01/2022

Cómo citar (APA, séptima edición):

Ruiz-Loayza, M. D., Valdiviezo-López, R., y Martínez-Quintanilla, R. F. (2022). Propuesta de modelo de gestión para disminuir la deserción de estudiantes de un instituto técnico superior de comunicaciones en el Perú. INNOVA Research Journal, 7(1), 1-18. https://doi.org/10.33890/innova.v7.n1.2022.1941

\section{Resumen}

Durante el año 2020 los institutos técnicos públicos y privados en el Perú, tuvieron un incremento importante en la deserción de sus estudiantes, como consecuencia del impacto de la pandemia Covid-19, el cual afectó el desempeño de sus estudiantes y la continuación de sus estudios. Esta realidad fue ocasionada por diversos factores: educacionales, psicológicos, económicos, sanitarios, entre otros; que requieren ser resueltas por las autoridades educativas, proponiendo soluciones efectivas para revertir la problemática de la deserción. La investigación tuvo como objetivo proponer modelo de gestión que permita disminuir la deserción estudiantil en un Instituto Técnico 
Superior de Comunicaciones. El estudio se planteó bajo un enfoque mixto, de nivel comprensivo y de tipo proyectiva, la cual incluyó como instrumentos una encuesta aplicada a 31 estudiantes y 4 entrevistas a colaboradores del instituto: administrador, secretario académico, docente y asistente administrativo. Los resultados de los principales hallazgos, muestran que el instituto requiere mejorar su servicio académico y administrativo, así como desarrollar los programas de estudios de manera adecuada. Asimismo, la investigación identifica alumnos con bajo nivel de formación educativa y desinterés por continuar sus estudios. Se concluye que es ineludible aplicar una gestión administrativa y pedagógica eficaz, implementando actividades de seguimiento, monitoreo y reforzamiento a los estudiantes, brindándoles apoyo psicológico de competencias y mejorando la gestión administrativa de la institución, a fin de disminuir la deserción de estudiantes de un instituto técnico superior de comunicaciones en el Perú.

Palabras claves: deserción; estudiantes; motivación; vocación; retención estudiantil.

\begin{abstract}
During the year 2020, public and private technical institutes in Peru, had a significant increase in the dropout rate of their students, because of the impact of the COVID-19 pandemic, which is affecting the performance of its students and the continuation of their studies. This reality was caused by various factors: educational, psychological, economic, health, among others; that need to be resolved by the educational authorities, proposing effective solutions to reverse the problem of dropout. The objective of the research was to propose a management model that allows to reduce student dropouts in a higher technical communications institute. The study was proposed under a mixed, comprehensive and projective approach, which included as instruments a survey applied to 31 students and 4 interviews with collaborators of the institute: administrator, academic secretary, teachers and administrative assistant. The results of the main findings show that the institute needs to improve its academic and administrative service, as well as to develop the study programs appropriately. Likewise, the research identifies students with a low level of educational training and lack of interest in continuing their studies. It's concluded that it is inescapable to apply an effective administrative and pedagogical management, implementing follow-up, monitoring and reinforcement activities for students, providing psychological support of competencies and improving the administrative management of the institution, in order to reduce the dropout rate of students from a higher technical communications institute in Peru.
\end{abstract}

Key words: dropout; students; motivation; vocation; student retention.

\title{
Introducción
}

La deserción de estudiantes se incrementó durante el año 2020, en los institutos técnicos públicos y privados en el Perú, como consecuencia del impacto de la pandemia Covid-19 y también ocasionada por diversos factores educacionales, psicológicos, económicos, sanitarios, entre otros, los cuales afectaron el desempeño académico de los estudiantes y que se manifestó en varios de los casos con la interrupción de sus estudios. Por dicha razón, la Organización de las Naciones Unidas para la Educación, la Ciencia y la Cultura señala que la pandemia ha generado interrupciones en la educación de alrededor de 800 millones de estudiantes en el mundo, comparativamente esto equivale a más de la mitad de la población mundial estudiantil (UNESCO, 2021). En esa medida, existen diversas investigaciones que plantean una serie de estrategias para disminuir la deserción de los estudiantes en las entidades educativas, teniendo sus autoridades el reto de evaluar y proponer estrategias efectivas para revertir la problemática de la deserción 
Propuesta de modelo de gestión para disminuir la deserción de estudiantes de un instituto técnico superior de comunicaciones en el Perú

estudiantil. Dichas estrategias deben estar orientadas a buscar alternativas que frenen la deserción e interrupción de los estudiantes, impulsando propuestas de mejora en la gestión administrativa, fortalecer la metodología educativa, implementar programas de apoyo motivacional a los alumnos, realizar evaluaciones de orientaciones vocacionales, asesorías, entre otras acciones importantes (Silva, 2018).

Según Ruiz (2018) como antecedente al problema local en el Perú, los resultados de su estudio demostró que el factor que influyó directamente y de manera decisiva en la deserción de los estudiantes, fue el tiempo invertido para llevar a cabo los estudios, así mismo, destacando otros factores como el socioeconómico, enfocado en el ingreso y estabilidad laboral; el factor institucional, relacionado con la atención y apoyo de la administración, y por último, el factor académico, referido a los planes y la metodología de enseñanza. La deserción es un problema complejo que está presente en la realidad educativa peruana, la cual está influenciada por diversos factores, que afectan en mayor o en menor medida su desempeño, especialmente a los estudiantes de bajos recursos económicos que tienen una limitada preparación educativa, siendo estas condiciones una barrera que le impide que éste pueda completar sus estudios y tenga motivaciones para culminar sus estudios técnicos o superiores. Complementando la problemática expuesta, también se confirmó a través de la investigación de Sánchez et al. (2017), que el incremento de la deserción es ocasionado por la baja calidad educativa que tienen muchos centros de enseñanza, que generan en sus alumnos desmotivación, insatisfacción y pérdida de interés, siendo importante y clave identificar, los principales factores que permitan pronosticar la retención de los estudiantes, a fin de contribuir a disminuir el índice de abandono.

Por otro lado, otras investigaciones nacionales también han analizado la influencia que tiene la gestión de la administración educativa con el abandono de los alumnos, Corzo (2018) demostró en su hipótesis de estudio, que la gestión se vincula con la deserción de los alumnos del IESTP Julio C. Tello de Huarochirí-Lima, teniendo como causas asociadas a los factores socioeconómicos, académicos e institucionales. Asimismo, Necochea, et al. (2017) evidenciaron en su investigación sobre deserción estudiantil para una escuela de medicina en Lambayeque en los años 2006-2014, que el abandono es más recurrente en mujeres (52,3\%), qué en varones, además, el total de abandono de la carrera en el primer año fue del 13,5\%, teniendo la promoción del 2013 un alto porcentaje de abandono (62,5\%), es decir, que solo el 26\% logró completar sus estudios. Los investigadores enfatizaron que los factores que incidieron en el abandono estudiantil fueron el rendimiento académico, la metodología educativa y la carencia económica; concluyendo que la frecuencia de abandono de los estudiantes en la escuela de medicina fue alta y que mayormente se presentó en las mujeres; siendo los causales con mayor relevancia la desaprobación de alguna materia y no estar de acuerdo con la metodología de enseñanza.

Anexado a lo anterior, Albornoz (2019) realizó una investigación sobre el abandono de los alumnos en las instituciones de educación superior del departamento de Junín, encontrando en su mayoría de casos a varones mayores de 25 años, que a través de los años (2014 al 2017) fueron incrementando en la deserción. Dentro de los motivos y causas de la renuncia a los estudios por parte de los alumnos, se identificó que tanto el sustento económico y la calidad de servicio que brinda la institución inciden sobre la deserción de dicho grupo de alumnos. Esta investigación logró descubrir que la causa principal del motivo del abandono es la insatisfacción que tienen los alumnos por el servicio que reciben de la institución educativa, sumando a ello otros componentes 
adicionales como los factores psicológicos, sociológicos y económicos. Se puede señalar que, en la mayoría de los casos de estudio, el problema de la deserción de los estudiantes no tiene un control eficiente de las condiciones y situaciones de los alumnos, observando carencias de estrategias efectivas que tiendan en anticiparse y disminuir la deserción de los alumnos.

El incremento de las deserciones de alumnos del instituto técnico superior de comunicaciones en el Perú, serían explicadas por los diversos factores que influyen en la realidad educativa, como son la situación económica del estudiante, la anticuada metodología académica que utilizan algunos docentes, la deficiente gestión administrativa de la institución, entre otras razones. Para Viera, et al. (2020) la deserción impacta en la sociedad en su conjunto, porque involucra aspectos económicos y sociales, que no solo repercuten sobre los alumnos, sino también en las familias, las instituciones educativas y sobre el desarrollo de nuestro país; dicha problemática influye sobre el sistema educativo, haciendo ineficiente tanto al Estado como a las propias instituciones de educación superior, al no conseguir el objetivo de lograr que los estudiantes completen el programa de estudios (Cayón et al., 2021). En ese sentido, la falta de una gestión administrativa y pedagógica en base a resultados, el limitado seguimiento y acompañamiento de las actividades estudiantiles y la falta de asesorías psicológicas para mejorar las competencias de los alumnos, conllevaría a que las autoridades educativas del instituto técnico superior, no logren un buen desempeño en la gestión administrativa y pedagógica, que coadyuve a disminuir el indicador de deserciones de estudiantes. Por ello, según (Fernández et al., 2014) citado en Viera et al. (2020), las organizaciones educativas de nivel superior se ven en la necesidad de desarrollar políticas para integrar los ámbitos académicos y sociales, bajo una perspectiva más particular del alumno, así como dentro de su contexto social, cultural y económico. A partir del conjunto de problemas indicados, la mejora de la gestión administrativa y pedagógica constituye el problema de investigación. Por dicha razón, es relevante conocer y evidenciar los factores que afectan sobre la deserción de los estudiantes de manera precisa, a fin de proponer mejoras viables que logren una gestión más eficaz.

La investigación tiene como objetivo general, proponer modelo de gestión administrativa y pedagógica, que permitan reducir la deserción estudiantil en un instituto técnico superior de comunicaciones en el Perú. Prosiguiendo en ese orden de ideas, los objetivos específicos fueron: (i) analizar la situación de la deserción estudiantil en un instituto técnico superior de comunicaciones y (ii) explicar los factores que inciden en la deserción estudiantil en un instituto técnico superior de comunicaciones.

\section{Marco teórico}

En relación a los fundamentos teóricos, la presente investigación se basó en la teoría de desarrollo organizacional fundamentada por Chiavenato (2006); Gareth (2008) y GarbanzoVargas (2016); quienes enfatizan que la base del desarrollo organizacional están conformados por el sistema organizacional, el nivel grupal y el nivel individual, destacando que el fin de toda organización es desarrollarse y posicionarse en un rubro del mercado, siendo necesario evaluar los factores internos como la administración, sus colaboradores, el marketing, la imagen, y los factores externos como la demanda y los cambios, con el propósito de mejorar y aumentar su efectividad. Dicha teoría señala que cada parte de la organización tiene un valor esencial donde cada uno debe asumir un rol, la fusión de estos debe permitir el desarrollo eficiente y eficaz de la organización. 
Propuesta de modelo de gestión para disminuir la deserción de estudiantes de un instituto técnico superior de comunicaciones en el Perú

Por ejemplo, integrar y alcanzar una sinergia en todos los eslabones de la organización, ayudará a enfrentar mejor los problemas que surjan en cualquiera de los ámbitos sea interno o externo, serán capaces de tener una mejor respuesta para plantear alternativas de solución que alcancen mayor eficiencia en la misma organización, consolidar a todas las áreas, proyectándose a un mismo objetivo para enfrentar cualquier cambio.

Asimismo, otro fundamento teórico del estudio de investigación fue la teoría del aprendizaje, a través del cual se valora las experiencias vividas como parte de nuestro aprendizaje, la teoría fundamentada a través de Schunck (2012), promueve que el estudiante alcance un desarrollo intelectual influido de los acontecimientos que cada día éste experimenta, siendo importante que los alumnos tengan un cambio conductual a partir del conocimiento adquirido. Las diversas teorías ofrecen alternativas estratégicas para que los estudiantes alcancen el objetivo de lograr culminar sus estudios, las mismas que deben hacerse efectivas con la intervención del docente. Los profesores deben preocuparse del aprendizaje de sus alumnos, buscando una mejor estrategia y alcanzar altos niveles de motivación en los estudiantes, con el propósito de fortalecer una respuesta adecuada a los estímulos del ambiente, orientándose para que éste pueda vincularse con su aprendizaje, obteniendo respuestas positivas ante cualquier problema que pueda presentarse y alcanzar sus logros (Gutiérrez, et al., 2018; Figueroa, et al., 2017).

Otra línea de investigación, es la teoría de retención de Tinto (1975) citado por Fonseca y García (2016), el autor considera que el grado de integración e interacción que presentan los estudiantes en la institución, tanto en el sistema educativo y social, explican en gran manera la permanencia o el abandono de los estudios por parte de los alumnos, debiendo de tener las instituciones la capacidad de mantenerlos matriculados en todo el desarrollo de la carrera elegida. Asimismo, según (England, 2012; Donoso y Schiefelbein, 2007; Fonseca y García, 2016; Torres, 2012), existen otros factores que influyen en la decisión de desertar, señalando, por ejemplo, el nivel de satisfacción con la educación que adquieren los alumnos, el apoyo y aliciente que puede tener de su familia, que en algunas ocasiones permite sobrellevar el débil apoyo académico que obtenga de las instituciones educativas. Todo este cúmulo de factores influye sobre las decisiones que tome el estudiante a lo largo de toda su carrera estudiantil, que en la mayoría de los casos donde exista deserción, será muy probable que no tuvo el soporte de la institución educativa.

Concerniente al marco conceptual, la deserción estudiantil es el abandono académico o el retiro fortuito y forzoso del alumno, que provoca a la entidad educativa una vacante difícil de ocupar, ocasionándole problemas financieros y desbalance económico. Se puede agrupar en dos tipos de deserción: la voluntaria referida a la renuncia del estudiante, y la involuntaria que se presenta como resultado de una decisión de la institución amparada en sus estatutos.

La probabilidad de abandono estudiantil se incrementa cuando los factores de influencia son negativos, tales como un rendimiento académico no satisfactorio, falta de integración social y la falta de compromiso institucional, según (Díaz, 2008; Suárez y Díaz, 2015; Viale, 2014, Saldaña y Barriga, 2010), los estudios de deserción se agrupan en cinco corrientes teóricas: en enfoque psicológico del fenómeno, el sociológico, el factor económico y la perspectiva institucional. La deserción puede estar relacionado a factores educacionales, institucionales, psicológicos, sociales, económicos, personales; es por ello, importante realizar el estudio de los factores que influyen para 
revertir la deserción y encaminar al estudiante mediante estrategias que logren concluir y cerrar un ciclo de una parte de sus vidas.

Respecto a la justificación teórica, el presente estudio se apoya en la teoría organizacional, la teoría del aprendizaje y la teoría de retención. De manera práctica, se justifica para una posterior aplicación en futuras investigaciones en el sector educativo tanto público y privado, relacionados con los factores de deserción de los estudiantes, haciendo de conocimiento a los empresarios, directores, gerentes generales de las entidades educativas, sobre los beneficios que puede alcanzar la aplicación de la gestión eficaz de estrategias que disminuyan la deserción de sus estudiantes, especialmente de los institutos técnicos o tecnológicos. Desde el punto de vista metodológico se justifica sobre el diseño de investigación mixta, de sintagma holístico, porque al integrar el enfoque y la información cuantitativa y cualitativa, se centra en hacer un diagnóstico de mayor profundidad del estudio, mediante la revisión de conceptos, metodologías y base teórica, pasando a la triangulación de la información, interpretando y realizando un análisis de los resultados, con el propósito de presentar a los gestores educativos que conducen la marcha de sus instituciones, aristas que fortalezcan aspectos de gestión deficiente en la filosofía de la deserción estudiantil, que presentan muchas instituciones de educación superior.

\section{Figura 1}

\section{Teorías relativas a la gestión eficaz para disminuir la deserción estudiantil}

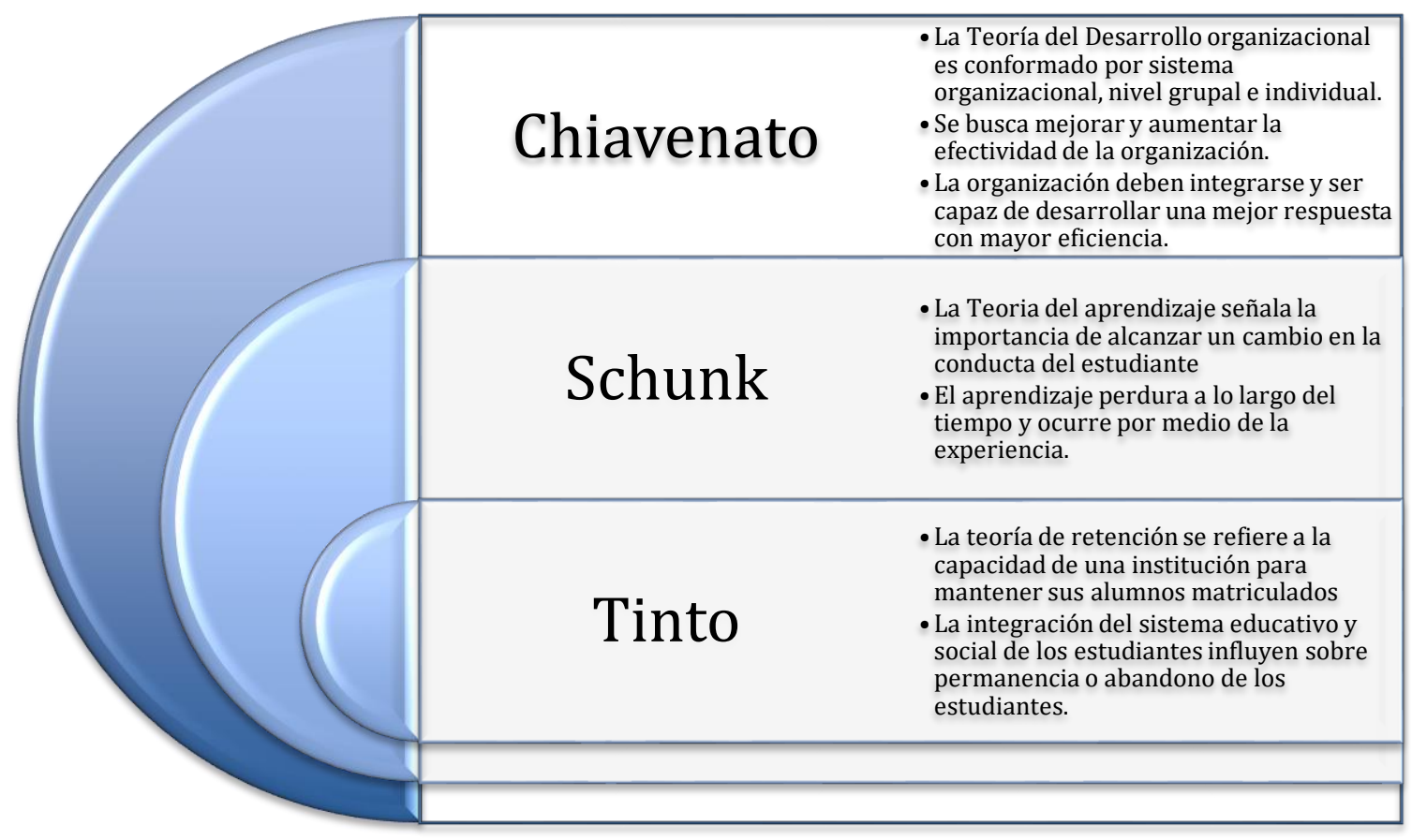

En la figura 1, se aprecia las teorías relativas a la gestión eficaz para disminuir la deserción estudiantil, en el marco de aplicación de estrategias para instituciones educativas. 
Propuesta de modelo de gestión para disminuir la deserción de estudiantes de un instituto técnico superior de comunicaciones en el Perú

\section{Metodología}

La investigación se sustenta en el enfoque mixto, sintagma holístico, la cual permite conceptualizar la complejidad del problema, integrando el enfoque cualitativo y cuantitativo (Carhuancho et al., 2019), mediante la aplicación de encuestas y entrevistas, lo cual amplía el estudio para identificar el problema y plantear propuesta de mejoras. Además, para alcanzar los resultados, se realizó el análisis de lo general a lo particular, bajo el método deductivo, el cual le faculta al investigador examinar los eventos de lo general a lo particular, apoyado a través de leyes, postulados, teorías y concluir en lo específico, para obtener soluciones a situaciones individuales (Naupas et al., 2013; Bernal, 2010).

En la recopilación de la información se contactó directamente a los encuestados, validando los instrumentos de medición para las siguientes subcategorías: deserción, estudiante y retención; primero se hizo una explicación breve de la encuesta, obteniendo un nivel de respuesta aceptable. En la investigación fue importante realizar la validación del instrumento, para asegurar la medición y cumplimiento de la finalidad en la investigación, en ese sentido, se validó el cuestionario a través de la opinión de tres expertos, corroborando que los ítems se relacionen con las dimensiones de manera efectiva, y a su vez, estas dimensiones se relacionen con las variables. Los profesionales suscribieron la ficha de validación con un puntaje del 100\%, obteniéndose las evidencias de validez por contenido con el coeficiente $\mathrm{V}$ de Aiken, a través del cual se ratificó la aplicación del mismo (Carhuancho, Nolazco, Sicheri, Guerrera y Casana, 2019; Peinado, 2015). Se aplicó una prueba piloto a 15 personas para medir los márgenes de error de los instrumentos y contar con la confiabilidad del mismo, para lo cual se utilizó el software SPSS, obteniendo el valor del Alfa de Cronbach de 0,90, determinando que el cuestionario tiene una alta confiabilidad, obteniéndose las evidencias alineadas con los datos del estudio.

En el presente estudio se tomó como población y muestra a 31 estudiantes del instituto superior técnico de comunicaciones; siendo la muestra la misma cantidad que la población (Hernández, Fernández, y Baptista, 2014). Las unidades informantes lo conformaron cuatro trabajadores de la institución que intervienen en el proceso de la gestión académica, el administrador, el secretario académico, un docente y un asistente administrativo, quienes están vinculados directamente con la gestión administrativa y académica del instituto superior. Se confeccionó un instrumento de recolección de datos, aplicando una encuesta con 14 preguntas enfocadas a las subcategorías: deserción, estudiante y retención. Asimismo, toda la información recopilada fue complementada mediante la observación para ampliar el análisis de la situación en estudio (Monje, 2011; Carhuancho et al., 2019). En la tabla 1 se presenta la matriz de las categorías y subcategorías aprioristas definidas en el presente estudio.

\section{Tabla 1}

Matriz de Categoría y Subcategorías: Deserción estudiantil

\begin{tabular}{ll}
\hline \multicolumn{1}{c}{ Subcategorías } & \multicolumn{1}{c}{ Indicadores } \\
\hline \multirow{3}{*}{ Deserción } & 1. Compromiso institucional \\
& 2. Integración social y económica \\
\hline
\end{tabular}

Esta obra se comparte bajo la licencia Creative Common Atribución-No Comercial 4.0 International (CC BY-NC 4.0) Revista de la Universidad Internacional del Ecuador. URL: https://www.uide.edu.ec/ 


\begin{tabular}{ll}
\hline \multicolumn{1}{c}{ Subcategorías } & \multicolumn{1}{c}{ Indicadores } \\
\hline Estudiante & 4. Vocación \\
Retención & 5. Persistencia \\
& 6. Motivación \\
\hline
\end{tabular}

\section{Resultados}

En los resultados cuantitativos de la encuesta realizada sobre 31 estudiantes del instituto, se encuentran algunas dificultades en la subcategoría deserción, donde el $45.1 \%$ de los estudiantes consideran que el factor económico siempre o casi siempre influyen en la decisión de continuar con sus estudios, mientras que el $29 \%$ de los alumnos indicó que la empatía en el entorno educativo en también un factor que influye sobre el abandono de los estudios, lo cual nos indica que existen deficiencias en la integración del alumno con la institución educativa. En la subcategoría estudiante al consultarles si la vocación del estudiante es un factor que influye para que abandone los estudios, el $45.1 \%$ indicó que siempre o casi siempre es una causa influyente, mientras que el $74.2 \%$ señalaron que la vocación por su carrera le permitirá siempre alcanzar sus objetivos profesionales. Por último, en la subcategoría retención se observa que el 51.6\% de estudiantes considera que a veces la relación afectiva con su familia puede influir en su rendimiento académico y/o retiro de la institución.

\section{Figura 1}

Diagrama de Pareto de la categoría deserción estudiantil

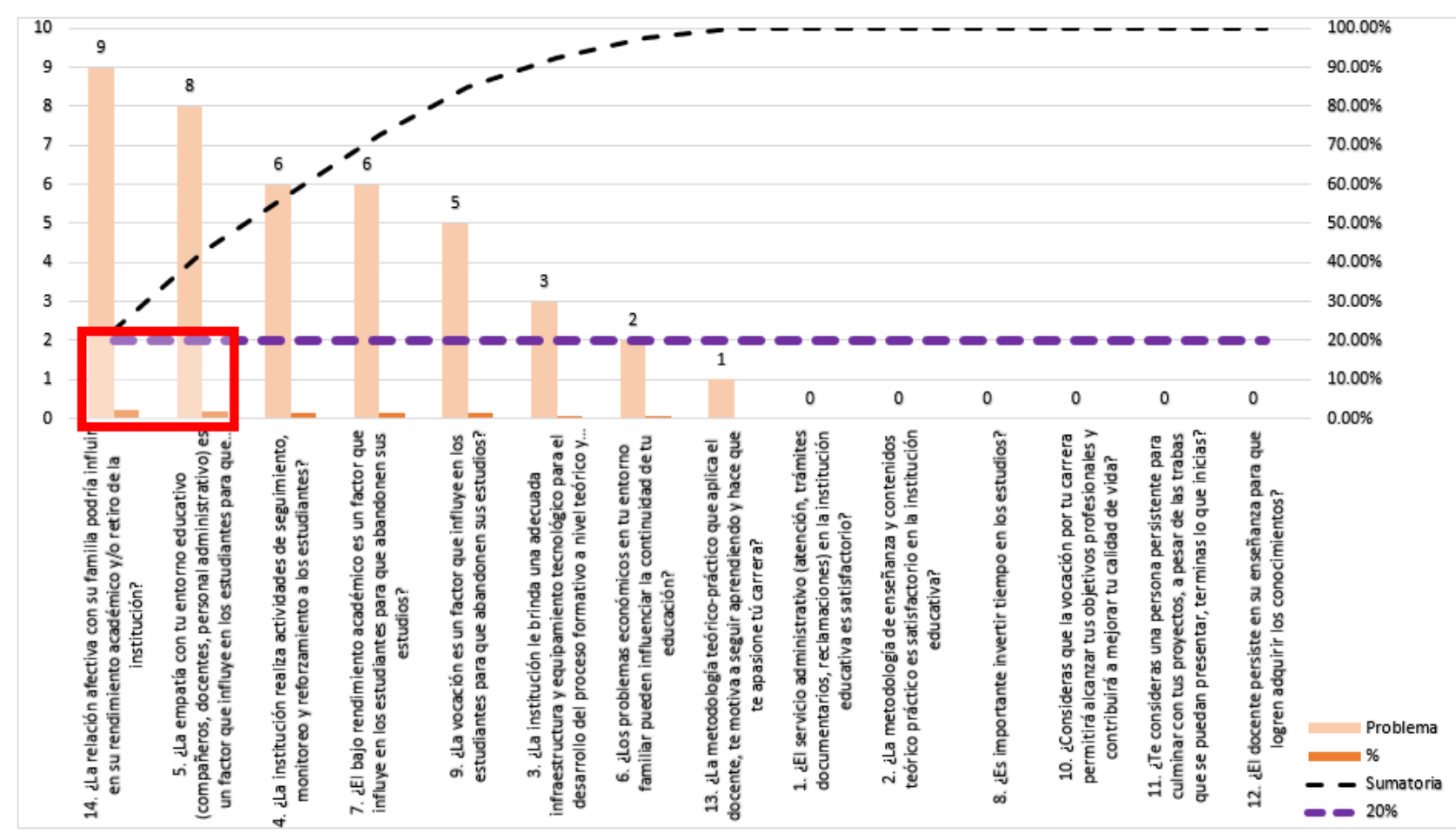

Fuente: Elaboración propia 
Propuesta de modelo de gestión para disminuir la deserción de estudiantes de un instituto técnico superior de comunicaciones en el Perú

En la figura 1 se muestra el análisis de Pareto, donde al lado izquierdo se puede observar la pregunta más crítica de la encuesta realizada, la cual es: ¿La relación afectiva con su familia podría influir en su rendimiento académico y/o retiro de la institución?; bajo este análisis se encontró que dicho punto equivale a un $22.50 \%$ en base al $20 \%$, demostrándonos que la familia es un factor que influye sobre el rendimiento académico y/o retiro del estudiante. En ese sentido, es necesario implementar estrategias para apoyar a los estudiantes con el manejo de sus emociones a fin que obtengan mayor seguridad en su vida profesional y personal.

A través de las encuestas se obtuvieron resultados que fueron ratificados posteriormente con las entrevistas, el sondeo coincide con el número de alumnos que desertaron en la institución en los últimos tres años, de acuerdo a sus registros administrativos en el ciclo 2020-I, el 57\% de alumnos desertó de sus estudios, siendo la mayor cantidad de estudiantes que abandonaron sus estudios, respecto al número de ingresantes, según se puede apreciar en la figura 2.

\section{Figura 2}

Frecuencia de deserción de estudiantes durante los tres últimos años

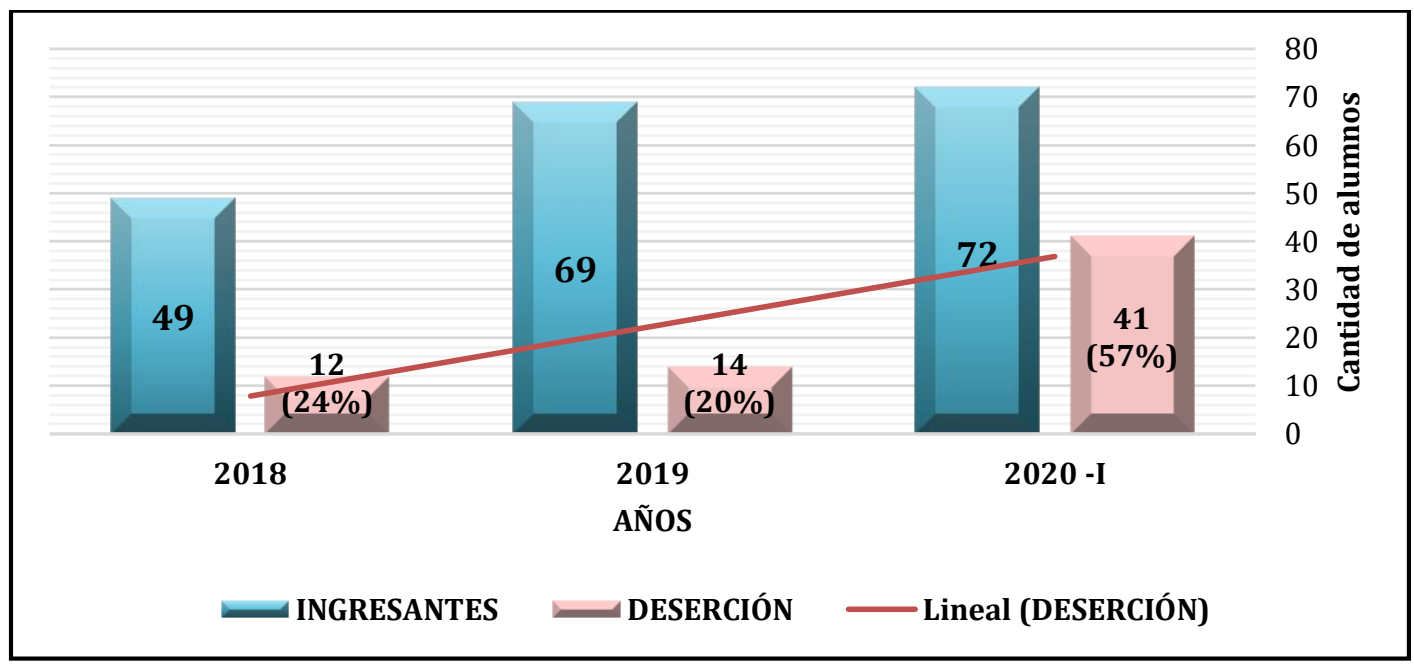

Fuente: Elaboración propia. 


\section{Análisis Cualitativo}

\section{Figura 3}

Análisis de la categoría deserción estudiantil

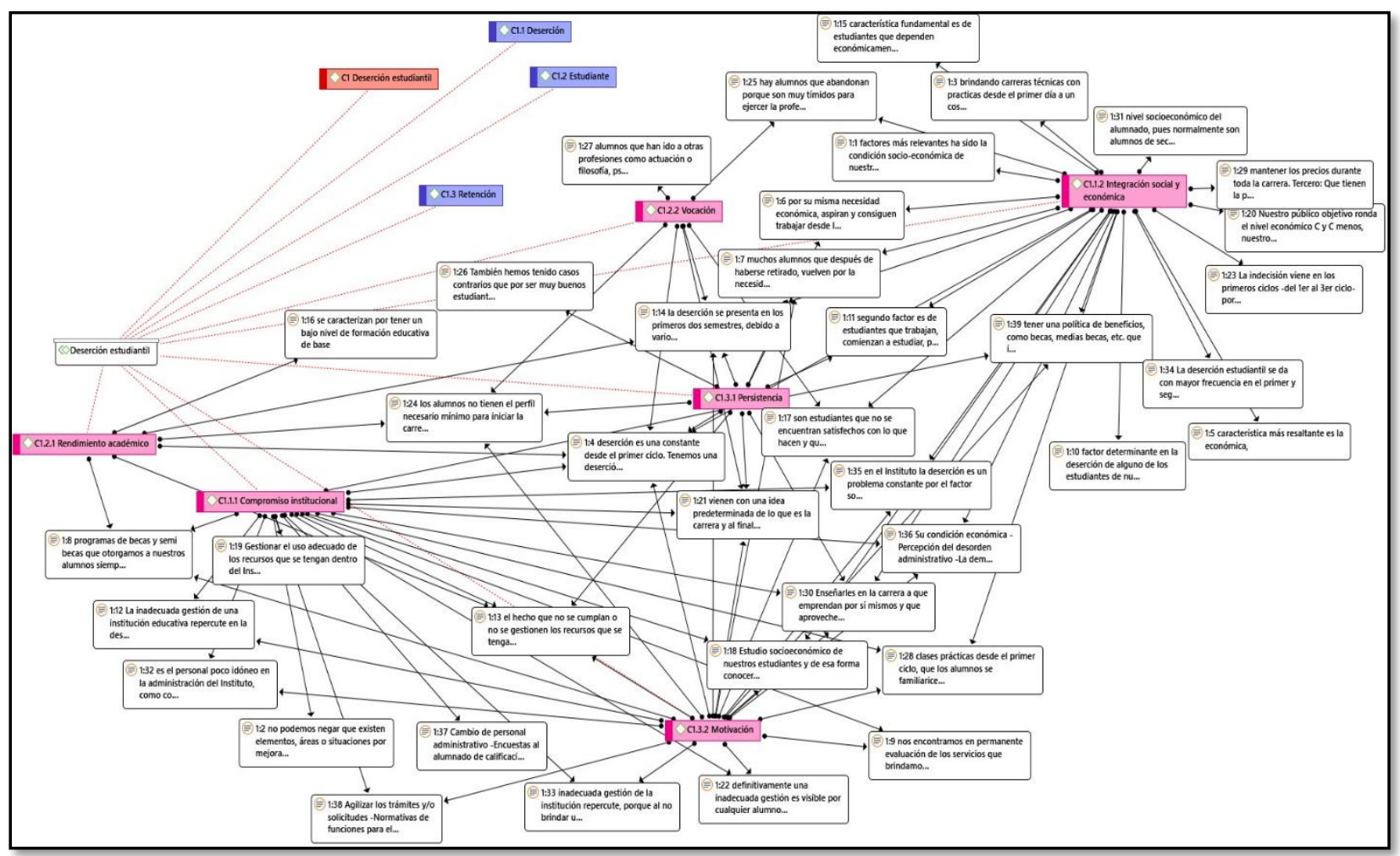

Fuente: Elaboración propia

En la figura 3, se observa el análisis cualitativo para la categoría deserción estudiantil, el cual presenta 3 subcategorías: deserción, estudiante y retención.

A través del análisis cualitativo se demostró que en las tres subcategorías presentan dificultades: la deserción, el estudiante y la retención; en la subcategoría deserción los entrevistados identificaron problemas relacionadas con el compromiso institucional y la integración social y económica de los estudiantes, consideran necesario mejorar y uniformizar la atención de los trámites del estudiante, ajustar la metodología de enseñanza para que llegue a todos, además de los que tienen buen rendimiento, buscando como propósito que todos los estudiantes estén satisfechos; inclusive aquellos que se encuentran en el nivel socioeconómico D, en donde se encuentran muchos alumnos con poca capacidad de comunicación. Para reducir el porcentaje de deserción se debe proponer optimizar el método de enseñanza, con un adecuado apoyo académico y de observación, implementando programas de inducción idóneos que sea suficientemente motivador, que refuerce y oriente mejor al estudiante (Valencia, 2016). 
Propuesta de modelo de gestión para disminuir la deserción de estudiantes de un instituto técnico superior de comunicaciones en el Perú

Figura 4

Análisis cualitativo de la subcategoría deserción

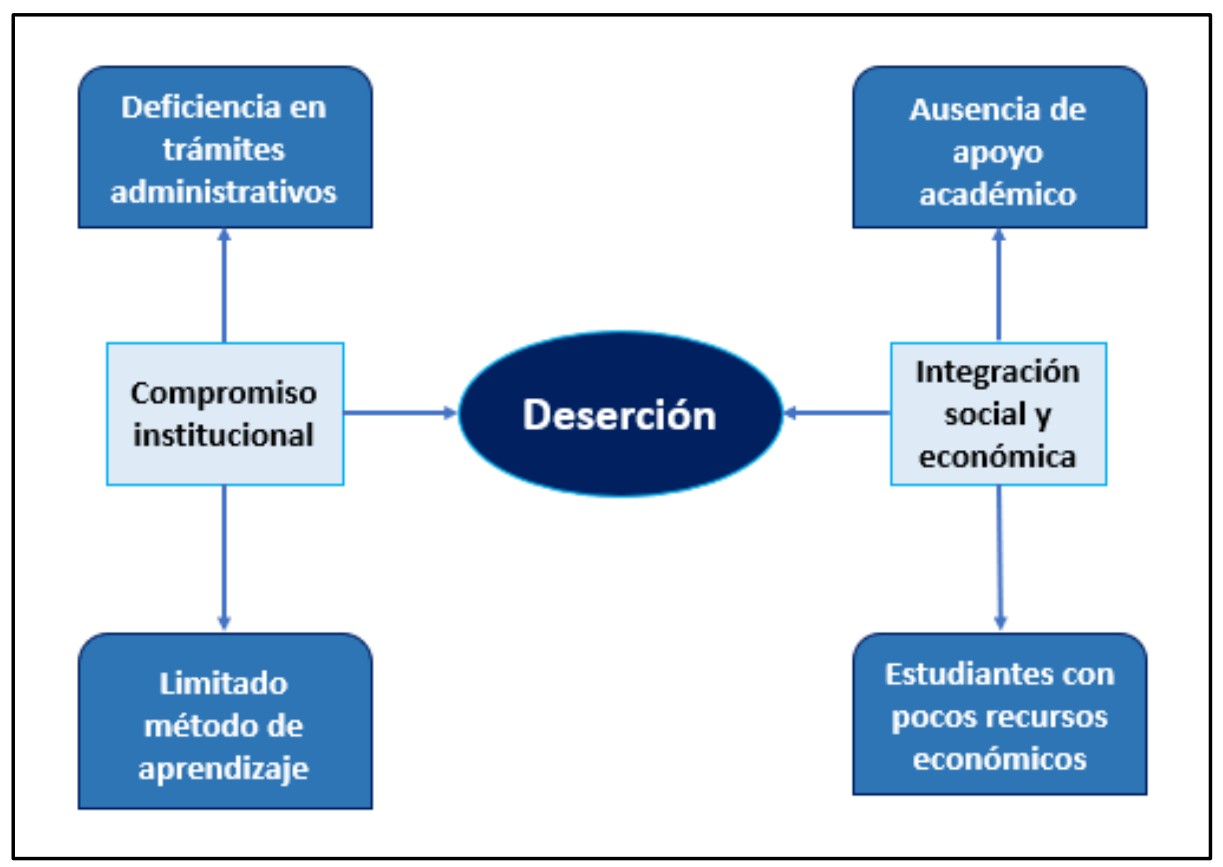

Fuente: Elaboración propia.

En la figura 4, se aprecia en el análisis cualitativo la subcategoría deserción, la cual contempla 2 indicadores: compromiso institucional e integración social y económica, conjuntamente con los principales problemas identificados en la triangulación.

En la subcategoría estudiante se establecieron dos indicadores: rendimiento académico y vocación, realizando un análisis cualitativo a partir de las entrevistas efectuadas, se identificaron estudiantes que presentaron un bajo nivel de formación educativa, el cual influyó sobre el buen rendimiento que obtuvieron en las notas de sus cursos, los resultados de su bajo desempeño, aunado a su precaria situación económica, les generó desmotivación, ocasionando que en algunos casos lo lleve a abandonar su carrera o prolonguen sus estudios mayores al tiempo previsto. En el caso de la vocación, los resultados cualitativos evidencian que hay estudiantes que no tienen el perfil necesario para estudiar la carrera que brinda el instituto, por no haber realizado una correcta orientación vocacional desde el inicio de la carrera, la cual hace imposible sostener su nivel de motivación y continuar su carrera, razón por la cual estos estudiantes pierdan el interés por los estudios y toman la decisión de abandonarlos, al no tener un soporte psicológico que lo reoriente.

El rendimiento académico es un factor de gran relevancia, que incide directamente en la deserción estudiantil, la desaprobación de algunas asignaturas y no estar de acuerdo con el método de enseñanza, son las causas de retiro más frecuentes, según Necochea, Nervi, Tuesta, Olazabal, Rodríguez, Gastelo y Leon (2017). 


\section{Figura 5}

\section{Análisis cualitativo de la subcategoría estudiante}

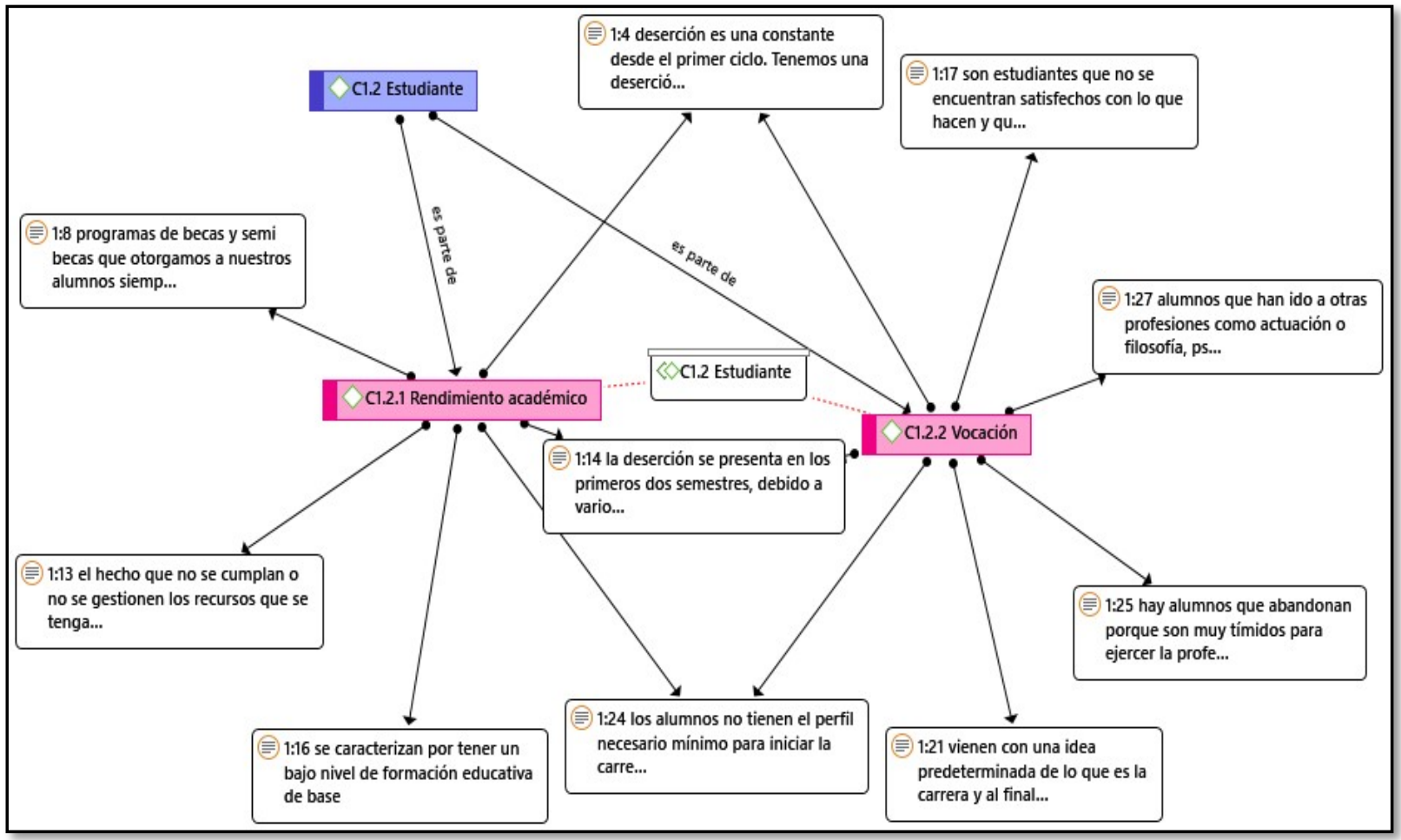

Fuente: Elaboración propia.

En la figura 5, se observa en el análisis cualitativo para la subcategoría estudiante, se presenta en 2 indicadores: rendimiento académico y vocación.

Por otro lado, en la subcategoría retención se definieron dos indicadores: persistencia y motivación, a través del análisis cualitativo de las entrevistas realizadas, se identificaron que muchos alumnos realizan un gran esfuerzo para culminar su carrera, existen alumnos que se ven en la necesidad de trabajar por necesidad económica, compartiendo sus actividades estudiantiles y laborales, asimismo, se tiene alumnos que después de haberse retirado vuelven a reincorporarse y continúan sus estudios, porque tienen claro su objetivo de contar con un título técnico profesional, siendo necesario que la institución promueva estrategias que brinde más oportunidades para la culminación de las carreras técnicas de sus alumnos.

En el análisis de la motivación de los alumnos, la encuesta ya había identificado la desmotivación que tenían algunos estudiantes por la gestión académica; en la entrevista a los gestores administrativos y al docente, en el análisis cualitativo del indicador motivación, también se recoge la percepción de una inadecuada gestión administrativa, debido a la existencia de un desorden administrativo y lentitud en la gestión de los trámites, los cuales son visibles por los propios alumnos. Si bien es cierto, la institución realiza algunas evaluaciones de la situación de los alumnos, buscando conocer el nivel de satisfacción del servicio brindado, la entidad es 
Propuesta de modelo de gestión para disminuir la deserción de estudiantes de un instituto técnico superior de comunicaciones en el Perú

consciente que las acciones de mejora son insuficientes, porque no se revierte la inadecuada gestión académica y administrativa.

En tal sentido, es imperativo establecer estrategias que logren objetivamente motivar a los alumnos, desde los métodos pedagógicos que deben ser mejorados por los docentes, una propuesta o alternativa sería implementar un departamento de psicología, que mejore la orientación vocacional de los alumnos, la institución debe lograr un mayor compromiso con todos sus alumnos, que permita incrementar el indicador de motivación. Para garantizar la permanencia de los estudiantes debe ofrecerse una calidad educacional óptima, que haga frente a los problemas que generan desmotivación, insatisfacción y pérdida del interés del estudiantado, de acuerdo a lo señalado por Sánchez, Barboza, y Castilla (2017).

\section{Figura 6}

\section{Análisis cualitativo de la subcategoría retención}

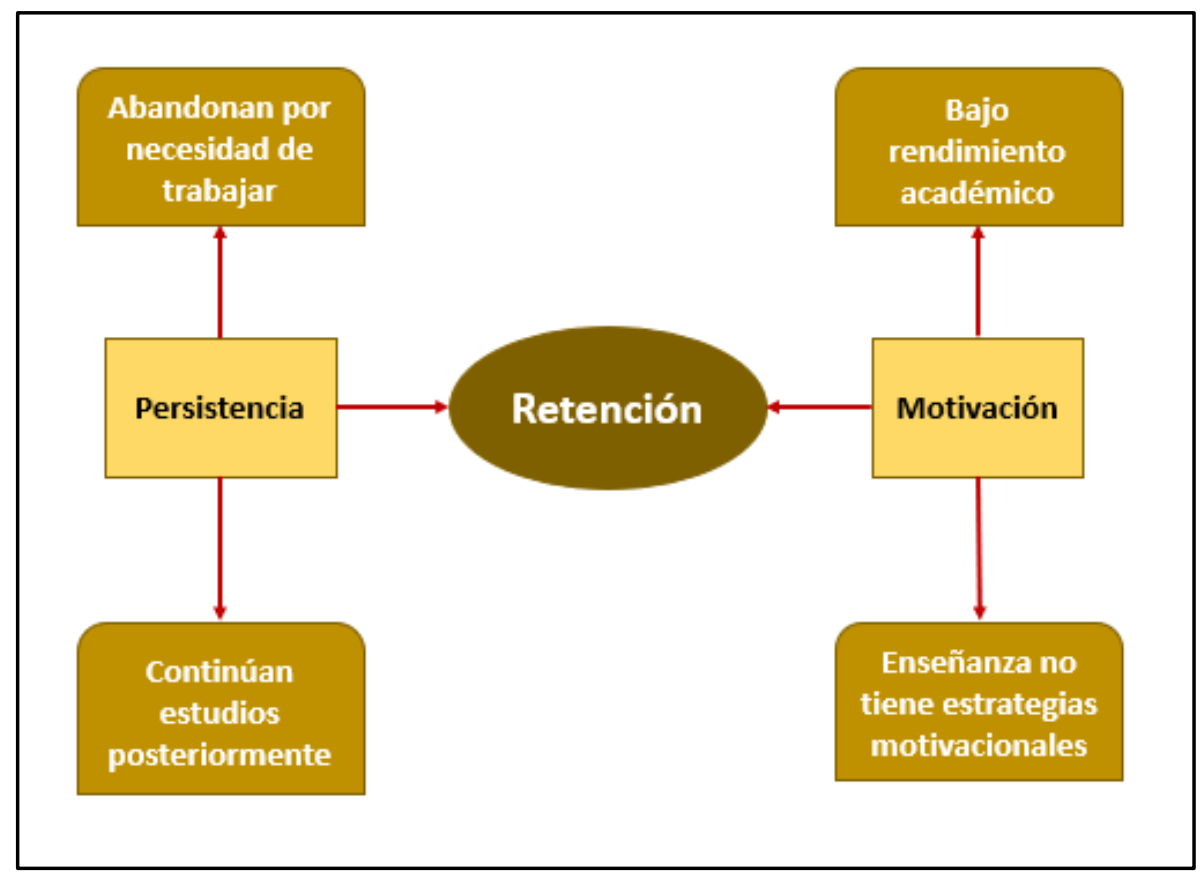

Fuente: Elaboración propia.

En la figura 6, se aprecia en el análisis cualitativo la subcategoría retención, la cual se respalda en 2 indicadores: persistencia y motivación, la cual describe los principales problemas identificados en la triangulación.

De acuerdo a los resultados del análisis cuantitativo y cualitativo, en base a la triangulación de los datos, se logró priorizar los siguientes problemas:

- Existe un limitado rendimiento académico del estudiante en el instituto. 
- Existe una inadecuada orientación vocacional de los estudiantes por no contar con el perfil necesario para estudiar la carrera.

- La Institución no cuenta con procedimientos establecidos para los trámites administrativos.

\section{Propuesta de modelo de gestión administrativa y pedagógica}

Basado en tres objetivos fundamentales:

Objetivo 1: Implementar actividades de seguimiento, monitoreo y reforzamiento a los estudiantes.

Objetivo 2: Brindar asesoría psicológica, de competencias que debe tener el estudiante según la carrera profesional elegida.

Objetivo 3: Mejorar la gestión administrativa de la institución.

\section{Figura 7}

Esquema de objetivos y tácticas del modelo de gestión

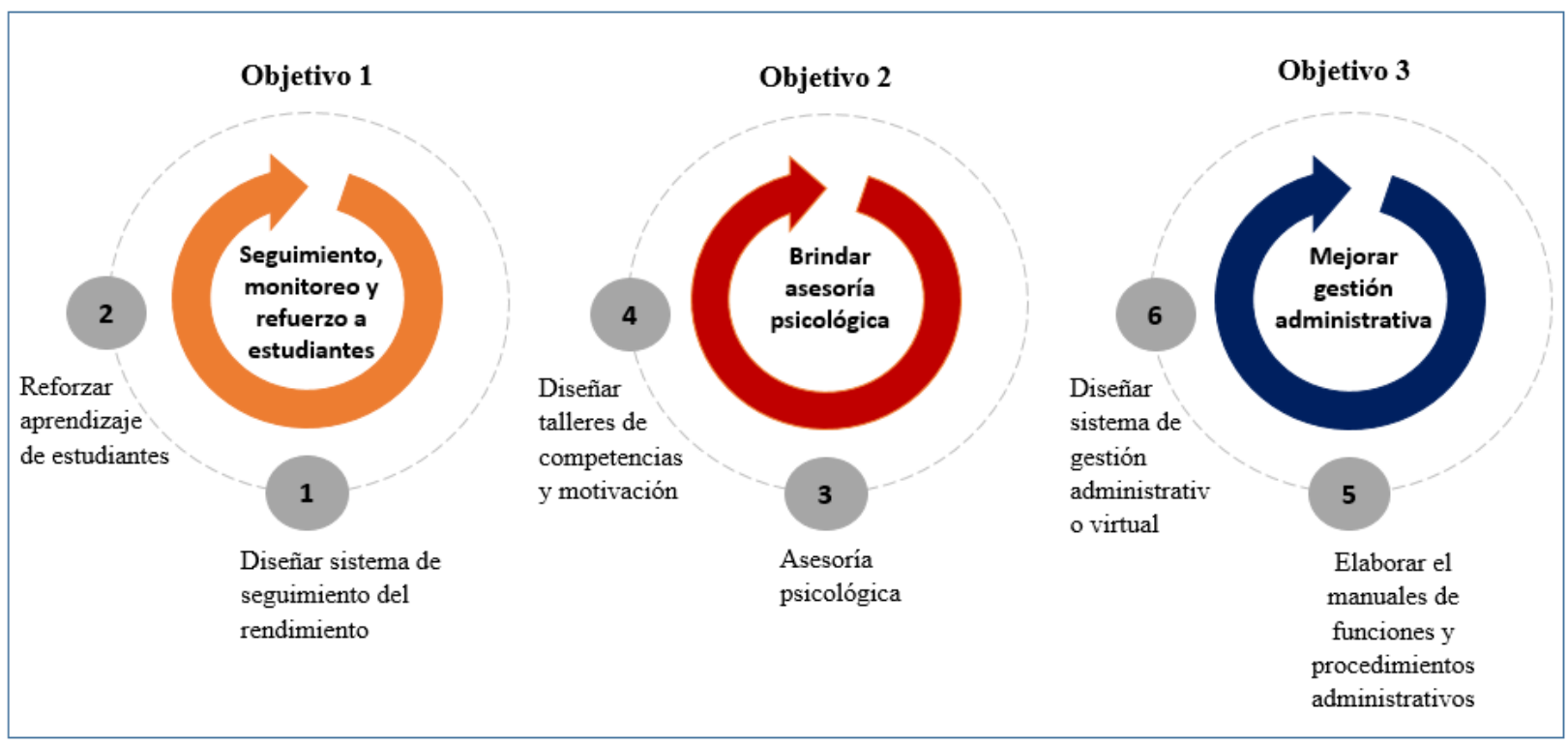

Fuente: Elaboración propia.

\section{Discusión}

Los resultados que se obtienen en la presente investigación titulada "Propuesta de modelo de gestión para disminuir la deserción de estudiantes de un instituto técnico superior de comunicaciones en el Perú", coinciden con los enfoques teóricos citados y relacionados con el desarrollo organizacional, del aprendizaje y retención. Dichas teorías plantean establecer estrategias para revertir la deserción, a través del incremento del nivel de preparación académica de los estudiantes, con programas de tutorías, orientación, asesoramiento e incentivándolos para 
Propuesta de modelo de gestión para disminuir la deserción de estudiantes de un instituto técnico superior de comunicaciones en el Perú

que los alumnos se esfuercen y les genere a ellos mismos beneficios potenciales, lo cual repercutirá con la disminución del riesgo de abandonar sus estudios, según (Ferreyra, Avitabile, Botero, Haimovich, y Urzúa, 2017).

Por otro lado, según los resultados, los estudiantes valoran de manera importante la relación afectiva con su familia, considerando que ésta influye sobre su rendimiento académico y posible abandono de sus estudios; esta valoración concilia con lo fundamentado por Bean y Metzner (1985) citado por Fonseca y García (2016), quienes señalan que los alumnos alentados por su familia, tienen más probabilidad de continuar sus estudios, a pesar de las dificultades e incertidumbre académica, porque compensan respecto al débil apoyo educativo que reciben de la institución.

En esa misma línea, la población en estudio, estuvo de acuerdo que la falta de integración y participación de los alumnos, son factores que influyen sobre el abandono de los estudios; lo mencionado por los estudiantes se comprueba a través de las teorías de (Bean y Eaton, 2001) que valoran la integración social y académica desde un punto de vista psicológico, citado por (Sánchez, Barboza, y Castilla, 2017), precisando que es de vital importancia que la institución educativa cuente con mecanismos, como grupos de aprendizaje y servicios de orientación personal y vocacional, a fin de integrar a los estudiantes a la institución. Si se logra implementar dichos mecanismos, se logrará la permanencia de los estudiantes que se encuentran en riesgo de desertar.

Asimismo, otro factor trascendental es la realización de seguimiento, monitoreo y reforzamiento a los estudiantes, la cual debe ser priorizada sobre aquellos que se encuentren en la posibilidad de desertar. Los resultados de la investigación tienen similitud con lo señalado por (Matheu et al., 2018; Zambrano et al., 2018), cuyos investigadores recomiendan que las entidades deben contar con formas de seguimiento o reforzamiento en el área académica, económica o psicoeducativa, sobre un conjunto de indicadores de mayor riesgo que influyen sobre la deserción de los alumnos, especialmente sobre aquellas que resulten con baja calificación, en la prueba de selección de ingreso de los estudiantes de una determinada carrera.

Finalmente, es necesario señalar que, para el estudio proyectivo se aplicaron las teorías de desarrollo organizacional, aprendizaje y retención de estudiantes, a través de la cual se elaboraron diversas estrategias y tácticas para la gestión eficaz del instituto técnico superior de comunicaciones, como el diseño de un sistema de seguimiento y monitoreo del rendimiento académico del estudiante, reforzamiento a los estudiantes para lograr un aprendizaje de las asignaturas, implementar asesorías psicológicas, diseñar talleres de competencias y motivacionales, así como de la implementación de un sistema de gestión administrativo virtual, con el diseño del manual de funciones, manual de los procedimientos para los trámites administrativos y del sistema informático de gestión administrativo. La aplicación y fortalecimiento de una gestión eficaz en el instituto técnico permitirá garantizar el éxito organizacional, siendo relevante contar con el compromiso de todos los actores involucrados, especialmente de la plana de la Dirección General, de las Jefaturas de las áreas administrativas y académicas, así como de los docentes, para lograr la disminución de la deserción de sus estudiantes. Dentro de la propuesta de esta investigación se recomienda que los próximos estudios apliquen el diseño experimental, para evaluar la eficacia que alcancen los institutos educativos con la aplicación metodológica de una gestión administrativa y pedagógica eficaz, de tal manera se 
contribuya al logro de los objetivos estratégicos de las instituciones educativas tanto técnicas y superiores.

\section{Conclusiones}

El objetivo de la investigación fue presentar un modelo de gestión que permita disminuir la deserción estudiantil en un instituto técnico superior de comunicaciones. Al respecto se definió como categoría de solución la propuesta de retención de Tinto para los estudiantes, el cual se orienta a mejorar el grado de integración e interacción de los alumnos en una institución educativa, para asegurar la permanencia en los estudios y la carrera elegida, proponiendo soluciones de mejora a los problemas diversos presentados y buscando la satisfacción de los estudiantes.

Para el desarrollo del diagnóstico de la investigación, se utilizaron instrumentos de recolección de datos cuantitativos y cualitativos, identificándose tres subcategorías resultantes, a partir de los conceptos de la categoría problema. Se evidenciaron tres problemas de mayor incidencia, el primero de ellos se relacionó con el limitado rendimiento académico del estudiante en el instituto. El segundo problema encontrado evidencia una inadecuada orientación vocacional de los estudiantes por no contar con el perfil necesario para estudiar la carrera. El tercer problema señala que la institución no cuenta con procedimientos establecidos para los trámites administrativos.

Con el propósito de cumplir con el objetivo principal de la investigación, se propuso solucionar los tres problemas encontrados, el primero de ellos, implementar actividades de seguimiento, monitoreo y reforzamiento a los estudiantes. El segundo, brindar asesoría psicológica, de competencias que debe contar el estudiante según la carrera profesional elegida. Finalmente, el tercer objetivo es mejorar la gestión administrativa de la institución.

A través del diagnóstico de la deserción estudiantil del alumnado del instituto técnico superior, se determinó que las subcategorías deserción, estudiante y retención presentan mayores dificultades. Se identificaron los factores que más influyen en la deserción estudiantil los cuales son el rendimiento académico, orientación vocacional y carencia de procedimientos administrativos.

En ese sentido, se recomienda adoptar la propuesta de solución del modelo de gestión para disminuir la deserción de estudiantes de un instituto técnico superior de comunicaciones en el Perú, a través de estrategias efectivas que reviertan el abandono de estudiantes, y se busque desarrollar una gestión administrativa y pedagógica más eficaz y eficiente en la institución educativa.

\section{Referencias Bibliográficas}

Albornoz, N. (2019). Análisis de la deserción estudiantil en institutos de educación superior tecnológicos durante el periodo 2014 - 2017, región Junín - Perú. [Tesis para obtener el grado de Maestra, Universidad César Vallejo, Junín, Perú]. https://bit.ly/2ZBRO1j

Bernal, C. (2010). Metodología de la investigación. Colombia. Pearson Educación. 
Propuesta de modelo de gestión para disminuir la deserción de estudiantes de un instituto técnico superior de comunicaciones en el Perú

Carhuancho, I., Sicheri, L., Nolazco, F., Labajos, F., Guerrero, M., Casana, K. (2019). Metodología de la investigación holística. Universidad Internacional del Ecuador -Guayaquil. https://repositorio.uide.edu.ec/handle/37000/3893

Cayón, E., Correa, J., y Scarpetta, L. (2021). Caracterización de los programas académicos y determinación de las variables que afectan el rendimiento de los estudiantes en el Colegio de Estudios Superiores de Administración, CESA. Formación universitaria, 14(5), 175186. https://dx.doi.org/10.4067/S0718-50062021000500175

Chiavenato, I (2006). Introducción a la Teoría General de la Administración. Vol. 7ma. Edición. Cuajimalpa: McGraw-Hill Interamericana.

Corzo, D. (2018). Gestión administrativa y deserción de estudiantes en el instituto Julio Cesar Tello del distrito de Huarochirí-Lima. [Tesis para grado de Maestra, Universidad César Vallejo, Lima, Perú]. https://bit.ly/3oiDJjH

Díaz, C. (2008). Modelo Conceptual para la Deserción Estudiantil Universitaria Chilena. Estudios Pedagógicos, 34(2), 65-86. http://doi.org/10.4067/S0718-07052008000200004

Donoso, S., y Schiefelbein E. (2007). Análisis de los Modelos Explicativos de Retención de estudiantes en la Universidad: una visión desde la desigualdad social. Estudios Pedagógicos, 33(1), 7-27. http://doi.org/10.4067/S0718-07052007000100001

England, C. (2012). Teoría Social Cognitiva y Teoría de Retención de Vincent Tinto: Marco Teórico para el estudio y medición de la autoeficacia académica en estudiantes universitarios. Griot. 5(1), 28-49. https://bit.ly/3ASAzXy

Ferreyra, M., Avitabile, C., Botero, J., Haimovich, F., Urzúa, S. (2017). At a Crossroads: Higher Education in Latin America and the Caribbean. Washington DC. World Bank Publications. http://hdl.handle.net/10986/26489

Figueroa, H., Muñoz, K., Vinício, E. y Zavala, D. (2017). Análisis crítico del conductismo y constructivismo, como teorías de aprendizaje en educación. Órbita Pedagógica 4, 1(12). https://bit.ly/2Zypw7W

Fonseca, G., y F. García (2016). Permanencia y abandono de estudios en estudiantes universitarios: un análisis desde la teoría organizacional. Revista de Educación Superior. RESU 45, 2539. https://bit.ly/3um $2 \mathrm{fBq}$

Garbanzo-Vargas, G. (2016). Desarrollo organizacional y los procesos de cambio en las instituciones. $\quad$ Revista Educación. http://doi.org/10.15517/revedu.v40i1.22534

Gareth, J. (2008). Teoría organizacional: Diseño y cambio en las organizaciones. Quinta Edición. México. Pearson Educación.

Gutiérrez, D., y otros (2018). Estrategias de aprendizaje: Una visión cognoscitivista. Universidad Pedagógica de Durango. http://www.upd.edu.mx/PDF/Libros/EstrategiasAprendizaje.pdf

Hernández, R., Fernández, C. y Baptista, M. (2014). Metodología de la Investigación. McGRAWHILL / Interamericana Editores S.A. de C.V.

Matheu, A., Ruff, C., Ruiz, C., Benites, L. y Morong, G. (2018). Modelo de predicción de la deserción estudiantil de primer año en la Universidad Bernardo O’Higgins. Educação e Pesquisa. 44. http://doi.org/10.1590/S1678-4634201844172094

Monje, C. (2011). Metodología de la investigación cuantitativa y cualitativa - Guía didáctica. Colombia. Universidad Surcolombiana. https://bit.ly/3oxR5Jh

Necochea, Y., y otros (2017). Frecuencia y características del abandono estudiantil en una Escuela de Medicina de Lambayeque, 2006-2014. Revista Médica Herediana 28(3), 171-177. https://doi.org/10.20453/rmh.v28i3.3184 
Ñaupas, H., Mejia, E., Novoa, E. y Villagómez, A. (2018). Metodología de la investigación cuantitativa - cualitativa y de redacción de la tesis. Colombia. Ediciones de la U. Quinta Edición. https://bit.ly/3F6S3SC

Ruiz, M. (2018). Factores que influyen en la deserción de los alumnos del primer ciclo de educación a distancia en la Escuela de Administración de la Universidad Señor de Sipán. Períodos académicos 2011-1 al 2013-1: lineamientos para disminuir la deserción. Educación, 27(52): 160-173. https://bit.ly/3oj3gt3

Saldaña, M., y Barriga, O. (2010). Adaptación del modelo de deserción universitaria de Tinto a la Universidad Católica de la Santísima Concepción, Chile. Revista de Ciencias Sociales (RCS) XVI, $\mathrm{n}^{\circ}$ 4, 616-628. https://www.redalyc.org/pdf/280/28016613005.pdf

Sánchez, G., Barboza, M. y Castilla, H. (2017). Análisis de la deserción y los factores asociados a la permanencia estudiantil en una universidad peruana. Actualidades Pedagógicas, $\mathrm{N}^{\circ}$ 69: 169-191. https://doi.org/10.19052/ap.4075

Schunk, D. (2012). Teorías del Aprendizaje. Una perspectiva educativa. México. Person Educación. Sexta Edición. https://bit.ly/3CYTMHu

Silva, R. (2018). Modelo Predictivo de Gestión Administrativa y deserción estudiantil en Programa de Pregrado adulto trabajador de Universidad Privada de Lima Metropolitana, año 2017. [Tesis para optar el grado Magister, Universidad Privada del Norte, Lima, Perú]. https://hdl.handle.net/11537/13723

Suárez, N., y Luz Díaz (2015). Estrés académico, deserción y estrategias de retención de estudiantes en la educación superior. Revista Salud Pública, 17(2), 300-313. http://doi.org/10.15446/rsap.v17n2.52891

Torres, L. (2012). Retención estudiantil en la educación superior. Revisión de la literatura y elementos de un modelo para el contexto colombiano. Colombia. Pontificia Universidad Javeriana. Primera Edición. https://bit.ly/3ihf634

Organización de las Naciones Unidas para la Educación, la Ciencia y la Cultura. (25 de enero de 2021). UNESCO revela una pérdida aproximada de $2 / 3$ de un año escolar en todo el mundo debido al COVID-19. https://tinyurl.com/kdarz8af

Valencia, C. (2016). Factores que inciden en la deserción estudiantil en el Programa de Administración de Empresas de una institución de educación superior en la ciudad de Bucaramanga. [Tesis para título de Mágister, Universidad Cooperativa de Colombia, Bucaramanga, Colombia]. https://bit.ly/3zSIClN

Viale, H. (2014). Una aproximación teórica a la deserción estudiantil universitaria. Revista Digital de Investigación en Docencia Universitaria 8(1), 59-75. https://doi.org/10.19083/ridu.8.366

Viera, D., Flores, M. y Pachari, E. (2020). Factores de deserción estudiantil. Un estudio exploratorio desde Perú. Interciencia: Revista de ciencia y tecnología de América, ISSN 0378-1844, 45(12), 586-591. https://dialnet.unirioja.es/servlet/articulo?codigo=7699995

Zambrano, G., Rodríguez, K. y Guevara, L. (2018). Análisis de la deserción estudiantil en las universidades del Ecuador y América Latina. Revista Pertinencia Académica, (8), 1-28. https://bit.ly/3m7RkI1 